\title{
Auxiliary Sources for the Near-to-Far-Field Transformation of Magnetic Near-Field Data
}

\author{
Vladimir Volski ${ }^{1}$, Guy A. E. Vandenbosch ${ }^{1}$, Davy Pissoort ${ }^{2}$ \\ ${ }^{1}$ ESAT-TELEMIC, KU Leuven, Leuven, Belgium, vladimir.volski@esat.kuleuven.be, guy.vandenbosch@esat.kuleuven.be \\ ${ }^{2}$ Reliability in Mechatronics and ICT, KU Leuven - Kulab, Ostend, Belgium, davy.pissoort@kuleuven.be
}

\begin{abstract}
A novel algorithm to perform a near-to-far-field transformation, starting from the magnetic field measured over a finite planar area above the device-under-test, is proposed. The algorithm is based on the introduction of auxiliary sources that approximate the magnetic field in the scanning plane outside but the scanning area. Numerical results show that these auxiliary sources significantly increase the accuracy of the near-to-far-field transformation.
\end{abstract}

Index Terms-Electromagnetic compatibility, Near-field, Radiation pattern.

\section{INTRODUCTION}

Radiation emissions generated by electronic devices are of interest in many areas. Nowadays almost every electronic device has to pass EMC radiated emission tests before it can be put onto the market. Standardized emissions tests are usually done in a semi-anechoic chamber and basically determine the far-field of the device-under-test. As such facilities are quite expensive to build, most companies must go to external labs to do these tests. This not only makes EMC testing quite expensive, but also limits the possibilities to efficiently debug an EMC issue. A more flexible and ofthen cheaper alternative approach is to measure in the very near field of the device-under-test and to perform a transformation between the near field measured data and the far field components. This technique was originally developed for antenna measurements, but later it was successfully used for other types of measurements. The equivalence theorem [1] provides a theoretical background for such transformations. Unfortunately, this theorem requires that the near field is known on a closed surface fully surrounding the radiator. In practice the scan data very rarely cover an entire closed surface and normally only the scan data above a device-undertest are available. As a consequence special algorithms are needed to cope with this. Most of the existing methods in literature [2-8] are based on the introduction of equivalent sources (mainly infinitesimal dipoles). These sources generate similar near field components over the scan area. The general idea actually leads to a classical inverse problem, which in many cases delivers non-unique solutions and involves operations with ill-conditioned matrices. In order to stabilize the solution several remedies already have been proposed in literature, involving special optimization algorithms with many regularization parameters, combinations with full wave solvers, etc. [2-8].

The main goal of this paper is to propose a simple flexible algorithm capable of predicting the level of radiation emission from a planar PCB based only on planar scan data over a final area and topological information about the board dimensions. The proposed algorithm is simple and relies on clear guidelines and well-known matrix operations only. Its implementation is straightforward. Moreover, in many cases this algorithm can be easily combined with other methods to improve the accuracy of the existing methods. The major difference with existing approaches found in literature is the fact that the auxiliary sources approximate the fields in the plane only outside the scan area and not over the scan area itself. It will be shown that this technique has huge advantages. In this paper we work out the theory for magnetic field components which are widely used in practice for EMI near field scanning [10].

\section{THEORY}

\section{A. Direct near to far field transformation}

Based on the equivalence theorem [1], the field outside a closed volume can uniquely be constructed from the electric and magnetic field components tangential to the surface of the volume. This theorem has three different versions, one where both electric and magnetic components are considered, one where only electric components are considered just in front of a magnetic conductor, and one where only magnetic components are considered just in front an electric conductor. Applying this general principle to the measurement plane, and using the second version, equivalent electric currents can be introduced based only on the magnetic near field components. In practice it is easier to measure magnetic field components. For a planar structure the equivalent electric current over a perfect magnetic conductor can be expressed in terms of a doubled electric current radiating in free space using the image principle [1]

$$
\mathbf{J}^{e}=2\left(\mathbf{i}_{z} \times \mathbf{H}\right)=-2 H_{y} \mathbf{i}_{x}+2 H_{x} \mathbf{i}_{y}
$$


Then the vector potentials can be introduce to calculate the far field components as

$$
A_{\alpha}^{e}=\frac{e^{-j k r}}{4 \pi r} \int_{V} J_{\alpha}^{e}\left(V^{\prime}\right) e^{j k\left(x^{\prime} \sin \theta \cos \theta+y^{\prime} \sin \theta \sin \varphi+z^{\prime} \cos \theta\right)} d V^{\prime}
$$

where $\alpha=x, y, k=2 \pi / \lambda, r=\sqrt{x^{2}+y^{2}+z^{2}}$

$A_{\theta}=A_{x} \cos \theta \cos \varphi+A_{y} \cos \theta \sin \varphi-A_{z} \sin \theta$

$A_{\varphi}=-A_{x} \sin \varphi+A_{y} \cos \varphi$

The vector potentials are coupled with the far field components as

$$
\begin{aligned}
& E_{\theta}=-j k W A_{\theta}^{e} \\
& E_{\varphi}=-j k W A_{\varphi}^{e}
\end{aligned}
$$

and $\mathrm{W} \approx 120 \pi$ is the free space impedance. These formulas (1)(4) are the core of the direct near-to-far-field transformation. In practice the scan area of a PCB of interest is always finite. As a consequence the equivalent currents (1) also have a finite domain. This restriction can result in huge errors even if the scan area is large. The analysis of (3) and (4) demonstrates that these equivalent currents (1) are e.g. not able to generate any theta far field components in the horizontal plane $\left(\theta=90^{\circ}\right)$. For typical antenna applications, electric currents generate directive radiation in the upper hemisphere. In this case the errors are less significant. However for a small PBC and especially at low frequencies, this far field approximation can be very inaccurate. As a consequence special measures should be taken to restore the correct emission from the PCBs.

\section{B. Asymptotic correction using auxiliary dipoles}

As we have shown in the previous section, a fundamental problem of any scanning technique is that the scan area is always limited. This can result in large errors because the contribution of the magnetic field outside the scan area is neglected. There are several possible ways to tackle this problem. In this paper an approach based on the introduction of auxiliary radiators ( $\mathbf{J}^{A R}$ ) is selected. This step looks very similar to other methods based also on the introduction of auxiliary sources. However there are two major differences. The original contribution from the equivalent currents (1) will be retained and the main goal of the auxiliary sources is to approximate the magnetic field components not over the scan area (as is normally done), but mainly outside it.

Consider a device under test (DUT) with electric currents $\mathbf{J}^{D U T}$ flowing on top PCB traces and a PCB ground plate marked by black solid lines in Fig. 1. Consider a finite planar scan area $S_{\text {scan }}$ in front of the DUT. The remaining part of the entire plane is denoted $S_{\text {out }}$.
Using the equivalence principle, the electric field above the scanning plane can be written in terms of the magnetic field in the scanning plane

$$
\mathbf{E}\left(\mathbf{J}^{D U T}\right)=\mathbf{E}\left(\mathbf{H}_{\text {scan }}\right)+\mathbf{E}\left(\mathbf{H}_{\text {out }}\right)
$$

Unfortunately, the second term in (5) is unavailable. For any auxiliary radiator $\mathbf{J}^{A R}$, the situation is different. It generates known field components everywhere in space, and thus also in the scanning plane, i.e. $\mathbf{H}_{\text {scan }}^{A R}$ and $\mathbf{H}_{\text {out }}^{A R}$. The use of the equivalence principle delivers an equation in which all terms are known.

$$
\mathbf{E}\left(\mathbf{J}^{A R}\right)=\mathbf{E}\left(\mathbf{H}_{\text {scan }}^{A R}\right)+\mathbf{E}\left(\mathbf{H}_{\text {out }}^{A R}\right)
$$

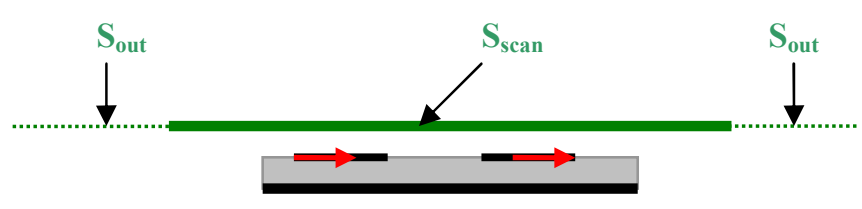

Figure 1. Introduction of auxiliary radiators.

Subtracting (6) from (5) and using the superposition principle, one obtains the following equation

$$
\begin{aligned}
\mathbf{E}\left(\mathbf{J}^{D U T}\right)=\mathbf{E}\left(\mathbf{J}^{A R}\right) & +\mathbf{E}\left(\mathbf{H}_{\text {scan }}-\mathbf{H}_{\text {scan }}^{A R}\right) \\
& +\mathbf{E}\left(\mathbf{H}_{\text {out }}-\mathbf{H}_{\text {out }}^{A R}\right)
\end{aligned}
$$

It becomes now obvious that if the auxiliary radiators are capable to mimic the field outside the scan area correctly then

$$
\mathbf{H}_{\text {out }}^{A R} \approx \mathbf{H}_{\text {out }}
$$

and (7) can be simplified

$$
\mathbf{E}\left(\mathbf{J}^{D U T}\right) \approx \mathbf{E}\left(\mathbf{J}^{A R}\right)+\left[\mathbf{E}\left(\mathbf{H}_{\text {scan }}\right)-\mathbf{E}\left(\mathbf{H}_{\text {scan }}^{A R}\right)\right]
$$

In this equation all terms in the right part are known. It is absolutely crucial to emphasize that in principle the procedure does not require a good approximation of the field $\mathbf{H}_{\text {scan }}$ by the field $\mathbf{H}_{\text {scan }}^{A R}$ inside the scan area as normally required by other methods. The approximation of the magnetic field components has to be of sufficient quality but in general this is a now simpler task because there are no hotspots or very fast variations of the fields outside the scan area. 


\section{Selection of auxiliary dipoles}

Infinitesimal electric dipoles are selected as auxiliary radiators. Groups of dipoles are able to mimic very complex topologies but the distance between adjacent dipoles should be chosen in a proper way. Guidelines used in the method of auxiliary sources [9] can be consulted. All field components generated by infinitesimal electric dipoles $\mathbf{J}^{e}$ can be calculated analytically

$\mathbf{H}\left(\mathbf{J}^{e}\right)=\left(-j k-\frac{1}{r}\right) \frac{e^{-j k r}}{r}\left[\begin{array}{l}\mathbf{i}_{y} \frac{z}{r}+\mathbf{i}_{z}\left(-\frac{y}{r}\right) \\ \mathbf{i}_{x}\left(-\frac{z}{r}\right)+\mathbf{i}_{z}\left(\frac{x}{r}\right) \\ \mathbf{i}_{x}\left(\frac{y}{r}\right)+\mathbf{i}_{y}\left(-\frac{x}{r}\right)\end{array}\right]^{T}\left[\begin{array}{c}J_{x}^{e} \\ J_{y}^{e} \\ J_{z}^{e}\end{array}\right]$

$E_{\alpha}=\frac{W}{j k}\left[\begin{array}{c}\delta_{\alpha \beta} k^{2}-Q_{\alpha \beta} k^{2}+\frac{j k 3 Q_{\alpha \beta}}{r} \\ +\frac{3 Q_{\alpha \beta}}{r^{2}}+\delta_{\alpha \beta}\left(-\frac{j k}{r}-\frac{1}{r^{2}}\right)\end{array}\right] \frac{e^{-j k r}}{4 \pi r} J_{\beta}$

$Q_{\alpha \beta}=\frac{x_{\alpha} x_{\beta}}{r^{2}} ; \quad \delta_{\alpha \beta}=1$ if $\alpha=\beta \quad \& \delta_{\alpha \beta}=0$ if $\alpha \neq \beta$

The positions and amplitudes of all auxiliary dipoles are defined in two steps. At first the positions and the polarizations of the dipoles are determined. The analysis of the scan data $\mathbf{H}_{\text {scan }}$ reveals several zones with high levels of measured magnetic fields. These zones cover all dominant sources of unwanted emission caused by the PCB traces. They can be easily formed by keeping all points from the scan data on the upland plateau around the local maxima. Then the equivalent horizontal dipoles are placed at the top of the PCB inside each zone

$$
\mathbf{J}_{H}^{\text {zone }}=i_{z} \times \mathbf{H}^{\text {zone }}, \quad z=z_{\text {top }}
$$

As we have already mentioned only horizontal dipoles are not able to predict correctly all far field components. So the presence of $\mathrm{z}$ directed auxiliary dipoles $J_{z}^{z o n e}$ is mandatory in many cases. In practice, the optimal positions of the $J_{z}^{z o n e}$ dipoles should coincide with vias supporting vertical currents and areas where vertical polarization currents in the substrate are large. Unfortunately, there are no simple ways to extract the required information from the near field scan data. As a consequence several approximations should be used. Vertical currents flowing on vias are transformed into horizontal currents spreading throughout horizontal traces. These horizontal currents generate clearly distinctive areas with a high field level in the scan data. So it is rather logical to assume that the vias are located within the zones already selected to cover dominant emission sources on the PCB traces. $J_{z}^{z o n e}$ dipoles are placed inside each zone using a simple equidistant grid. In contrast to the $J_{H}^{z o n e}$ dipoles which can belong to the same group within each zone, the $J_{z}^{z o n e}$ dipoles are independent within each zone because the exact positions of the vias are unknown. The combination of both dipole types ( $\left.J_{H}^{z o n e}, J_{z}^{z o n e}\right)$ forms a first level approximation. The magnetic field outside the scan area in the horizontal plane can be described as a superposition of outgoing waves generated by the real electric currents. The auxiliary dipoles are also generating outgoing waves there. These two sets of waves are equated in a sufficient number of points at the edges of the scan area, resulting in an $m \times n$ linear equation system. The following equations are used for the $k$-th point on the edge

$$
\begin{aligned}
& H_{x k}\left(J_{H}^{z o n e}\right)+H_{x k}\left(J_{z}^{z o n e}\right)=H_{x k}^{s c a n} \\
& H_{y k}\left(J_{H}^{z o n e}\right)+H_{y k}^{d i p}\left(J_{z}^{z o n e}\right)=H_{y k}^{s c a n}
\end{aligned}
$$

The obtained system (13) can be solved using the Gauss Legendre least square error minimum algorithm. The solution in matrix form is

$$
\mathbf{A J}=\mathbf{B} \rightarrow \mathbf{A}^{\mathbf{T}} \mathbf{A} \mathbf{J}=\mathbf{A}^{\mathbf{T}} \mathbf{B} \rightarrow \mathbf{J}=\left(\mathbf{A}^{\mathbf{T}} \mathbf{A}\right)^{-1} \mathbf{A}^{\mathbf{T}} \mathbf{B}
$$

Once the amplitudes of the auxiliary dipoles are calculated using (14), all far field components can be found using (9).

\section{NUMERICAL RESULTS}

In order to illustrate our method we consider a simple PCB with several traces on the top as shown in Fig. 2. The PCB thickness is $1 \mathrm{~mm}$ and its size is $120 \mathrm{~mm} \times 80 \mathrm{~mm}$. The distribution of magnetic field components above the PCB at a height of $2 \mathrm{~mm}$ at $200 \mathrm{MHz}$ was calculated using the FDTD solver that is included in Agilent Technologies' 3D EM platform EMPro [11]. The scan area is $16 \mathrm{~cm} \mathrm{x} 12 \mathrm{~cm}(0.107 \lambda$ $\mathrm{x} 0.08 \lambda$ ) and the used resolution is $0.5 \mathrm{~mm}$ in both directions.

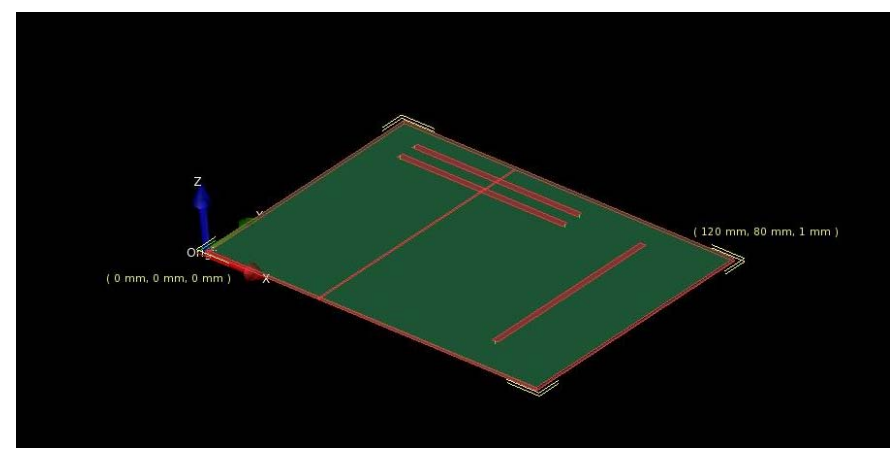

Figure 2. Screenshot of the PCB test structure with multiple traces

The combined reference magnetic field components are shown in Fig. 3. As expected the field level becomes very high above the PCB traces. 


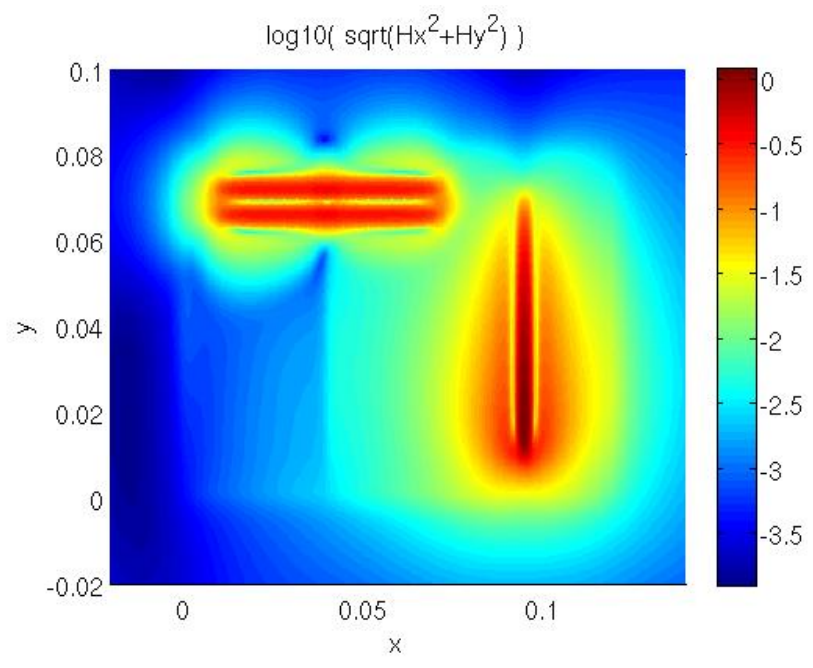

Figure 3. Calculated $x$ component of magnetic field over the scan area

Auxiliary dipoles are placed inside each zone using an equidistant grid. Its resolution is different from the resolution of the scan area. In this paper, the distance between adjacent dipoles is selected as $s_{d i p}=0.01 \lambda=15 \mathrm{~mm}$. For the structure in

Fig. 2 the total number of auxiliary dipoles is 24 and their positions are shown in Fig. 4. They form 16 groups (unknowns) containing several or single dipoles. The test points at the edges are also selected using an equidistant grid $\left(s_{\text {edge }}=0.01 \lambda=15 \mathrm{~mm}\right)$.

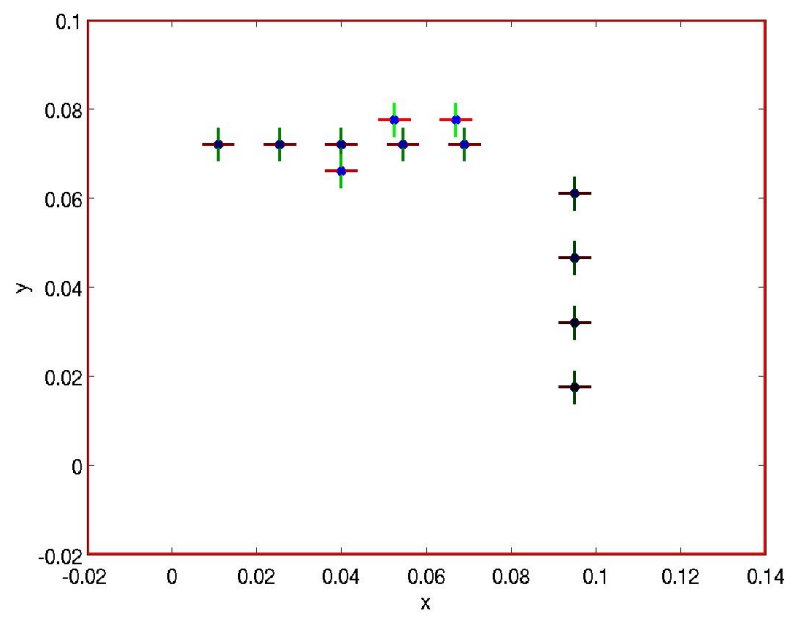

Figure 4. Original positions of all auxiliary dipoles

As we have already mentioned the imposed boundary condition is satisfied at the edges of the scan area. The least square errors minimum algorithm is used. The efficiency of the approximation can be checked once the amplitudes of all dipoles are calculated. The two magnetic field components at the edges are plotted in Fig. 5. As expected, a few dipole groups are capable to generate almost the same field at the edges of the scan area. There are small differences because the number of equations is different from the number of unknowns.
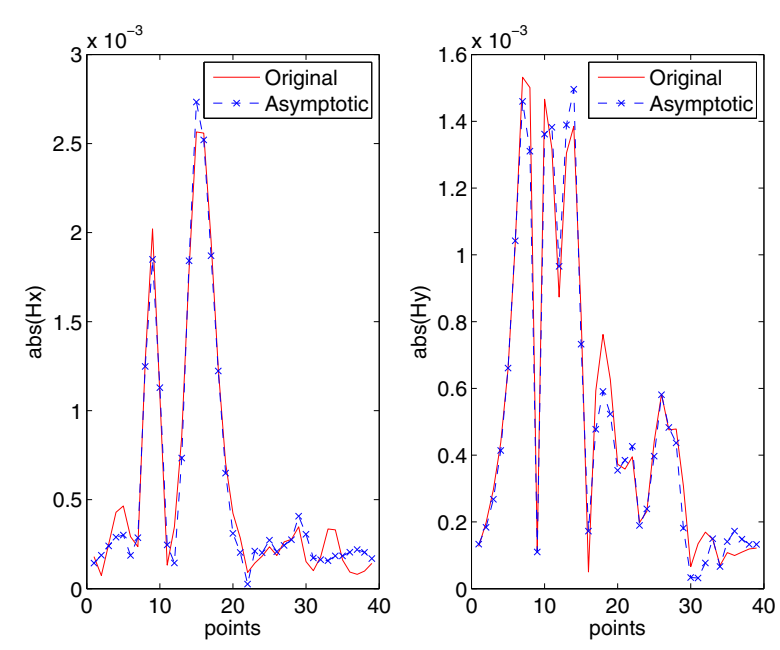

Figure 5. Boundary conditions for magnetic field components

The reference far field patterns and two calculated patterns are plotted in Fig. 6. The figures in the first column correspond to the reference patterns. The second column shows the results obtained using only the direct transformation of the near field scan data. The last column illustrates the correction introduced by our approach. As already mentioned the equivalent currents are not able to mimic the theta component $\left(E_{\theta}\right)$ of the far field pattern closer to the horizontal plane because they generate no radiation in these directions. This is a serious disadvantage of a small scan area. However, the other components $\left(E_{\varphi}\right)$ of the far field pattern are approximated satisfactorily. The application of our method allows to improve noticeably the approximation of the far field patterns in all angular sectors using just a few dipoles. This example illustrates the efficiency of our approach.

\section{CONCLUSIONS}

In contrast to many existing algorithms the approach introduced in this paper is very simple and straightforward. It relies on clear guidelines for auxiliary dipoles and simple operations with matrices. Moreover, the basic idea can be easily implemented in any other method using auxiliary dipoles. It significantly improves the accuracy of the direct approximation for all far field angular sectors as shown in Fig. 6. A future work will be focused on an advanced algorithm for the optimal placement of auxiliary dipoles and special measures to avoid possible problems caused by the operation with ill-conditioned matrices.

\section{REFERENCES}

[1] C. Balanis, "Antenna theory: analysis and design", Wiley, 1997

[2] X. Tong, D.W.P. Thomas, A. Nothofer, and P. Sewell, C. Christopoulos, "Modeling Electromagnetic Emissions From Printed Circuit Boards in Closed Environments Using Equivalent Dipoles", IEEE Transactions on Electromagnetic Compatibility, vol. 52, pp. 462470,2010 
[3] Z.W. Yu, J.A. Mix, S. Sajuyigbe, K.P. Slattery, and J. Fan, "An Improved Dipole-Moment Model Based on Near-Field Scanning for Characterizing Near-Field Coupling and Far-Field Radiation From an IC" , IEEE Transactions nn Electromagnetic Compatibility, Volume: 55 Issue: 1 pp. 97-108, 2013

[4] Y. Alvarez, F. Las-Heras, and M.R. Pino, "Reconstruction of equivalent currents distribution over arbitrary three-dimensional surfaces based on integral equation algorithms", IEEE Trans Antennas Propagation, Volume: 55 Issue: 12, pp. 3460-3468, 2007

[5] H. Weng, D.G. Beetner, and R.E. DuBroff, "Prediction of Radiated Emissions Using Near-Field Measurements", IEEE Transactions on Electromagnetic Compatibility, Volume: 53, Issue: 4, pp. 891-899, 2011

[6] Y. Vives-Gilabert, C. Arcambal, A. Louis, P. Eudeline, and B. Mazari, "Modeling Magnetic Emissions Combining Image Processing and an Optimization Algorithm", IEEE Transactions on Electromagnetic Compatibility, vol. 51, No.4, pp. 909-917, 2009

[7] Y. Vives-Gilabert, C. Arcambal, A. Louis, F. de Daran, and P. Mazari, "Modeling magnetic Radiations of electronic circuits using near-field scanning method", IEEE Transactions on Electromagnetic Compatibility, vol. 49, No.2, pp. 391-400, 2007

[8] P.F. López, C. Arcambal, D. Baudry, S. Verdeyme, and B Mazari, "Simple Electromagnetic Modeling Procedure: From Near-Field Measurements to Commercial Electromagnetic Simulation Tool", IEEE Transactions on Instrumentation \& Measurement, Volume 59, Issue 12, pp.3111 3121, 2010

[9] D.I. Kaklamani and H.T. Anastassiu, "Aspects of the Method of Auxiliary Sources (MAS) in computational electromagnetics" , IEEE Antennas \& Propagation Magazine, Volume 44, Issue 3, pp. 48-64, 2002

[10] T. Dorne, F. Vanhee, T. Grenson, D. Pissoort, D. Deschrijver, I. Couckoyt, T. Dhaene, " Optimized Sequential Sampling Algorithm for EMI Near Field Scanning", Proc. of the 2013 International Symposium on Electromagnetic Compatibility (EMC Europe 2013), Brugge, Belgium, September 2-6, 2013

[11] Agilent Technologies, Agilent EMPro, available from: http://www.home.agilent.com/
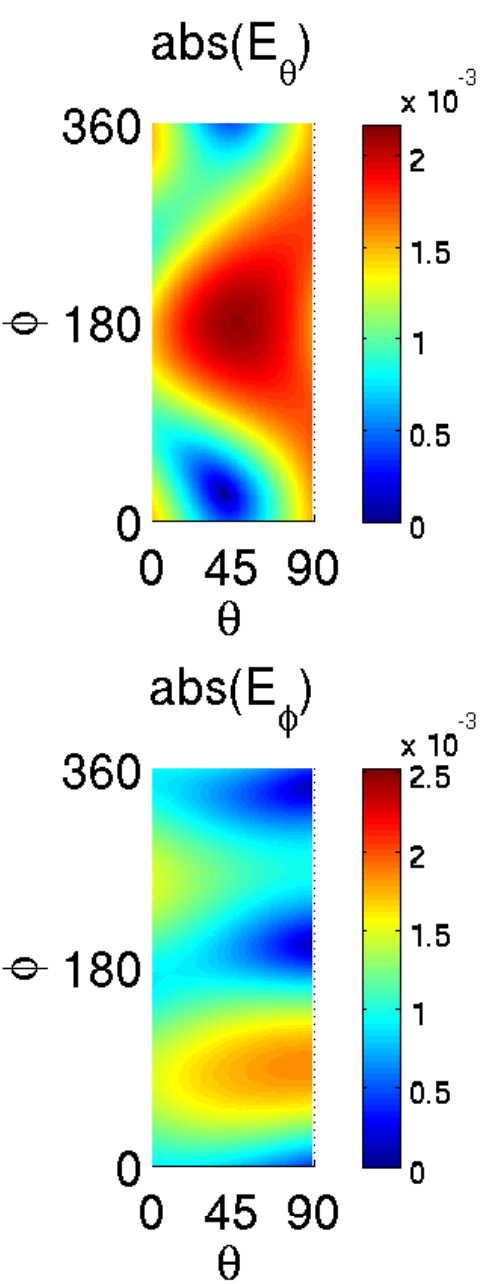
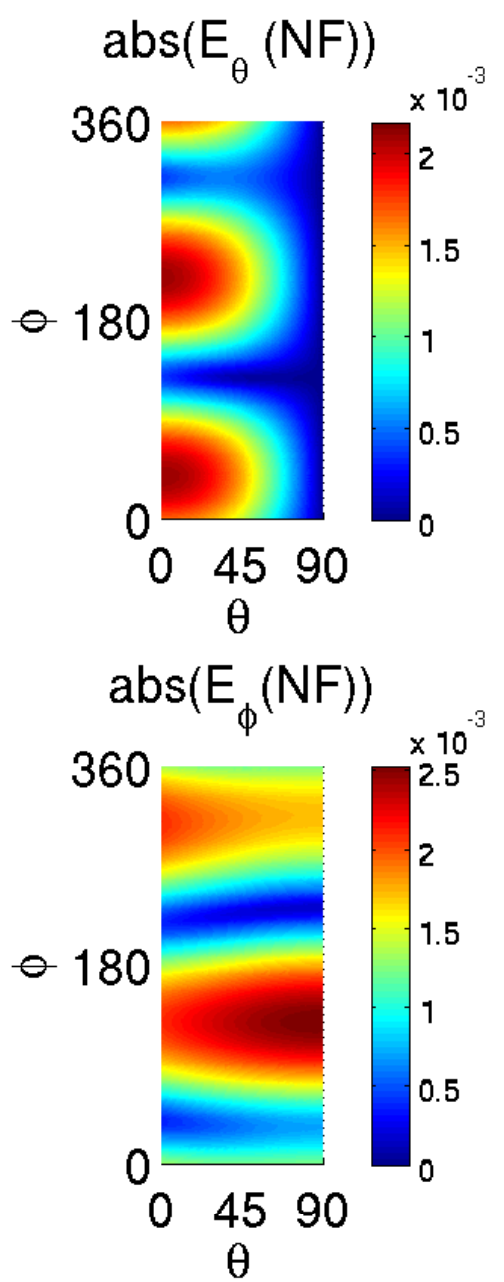
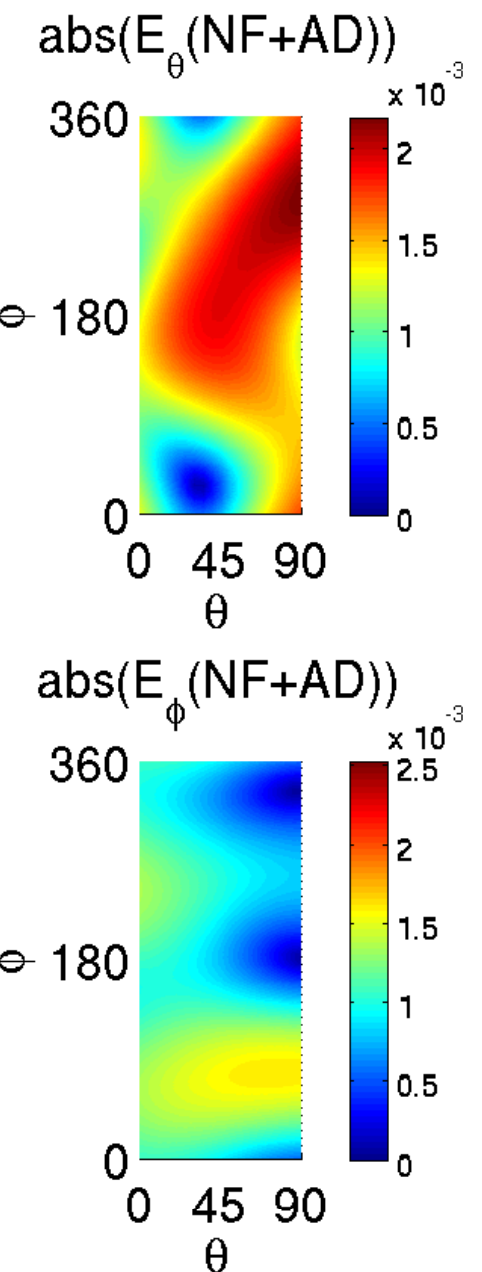

Figure 6. Reference and calculated far field patterns 